\title{
Homotopy Analysis Method for Boundary-Value Problem of Turbo Warrant Pricing under Stochastic Volatility
}

\author{
Hoi Ying Wong ${ }^{1}$ and Mei Choi Chiu ${ }^{2}$ \\ ${ }^{1}$ Department of Statistics, The Chinese University of Hong Kong, Hong Kong \\ ${ }^{2}$ Department of Mathematics and Information Technology, Hong Kong Institute of Education, Hong Kong \\ Correspondence should be addressed to Hoi Ying Wong; hywong@cuhk.edu.hk
}

Received 13 December 2012; Accepted 30 January 2013

Academic Editor: Bashir Ahmad

Copyright (c) 2013 H. Y. Wong and M. C. Chiu. This is an open access article distributed under the Creative Commons Attribution License, which permits unrestricted use, distribution, and reproduction in any medium, provided the original work is properly cited.

Turbo warrants are liquidly traded financial derivative securities in over-the-counter and exchange markets in Asia and Europe. The structure of turbo warrants is similar to barrier options, but a lookback rebate will be paid if the barrier is crossed by the underlying asset price. Therefore, the turbo warrant price satisfies a partial differential equation (PDE) with a boundary condition that depends on another boundary-value problem (BVP) of PDE. Due to the highly complicated structure of turbo warrants, their valuation presents a challenging problem in the field of financial mathematics. This paper applies the homotopy analysis method to construct an analytic pricing formula for turbo warrants under stochastic volatility in a PDE framework.

\section{Introduction}

Turbo warrant first appears in Europe but is now available under various names in many markets including the UK, Germany, Switzerland, Italy, Australia, New Zealand, Singapore, South Africa, Taiwan, and Hong Kong. For instance, it is called turbo warrant in the Nordic Growth market, contract for difference $(\mathrm{CFD})$ in the UK, and callable bull/bear contract $(\mathrm{CBBC})$ in Hong Kong. According to a report by Hong Kong Exchanges and Clearing Limited (HKEx) in 2009 [1], the UK has a big OTC market for CFDs while Hong Kong has a big exchange market for CBBC. Typically, HKEx listed 1,525 CBBCs, constituting $28 \%$ of the total number of its listed securities, and the total issued amount reached HK\$704 billion or US\$95 billion at the end of May 2009 [1]. Hong Kong has two types of CBBC: $\mathrm{N}$ and $\mathrm{R}$. The N-CBBC pays no rebate when a preselected barrier is crossed whereas the RCBBC pays a lookback rebate. If the barrier is not breached by the underlying asset price during the life of the contract, then the turbo call (put) is equivalent to the standard call (put). Therefore, turbo warrant resembles a combination of barrier and lookback options.

A comprehensive mathematical treatment of turbo warrants is given in [2], which provides several model-free properties of turbo warrants. This leads to subsequent extensions to turbo warrant pricing with jump-diffusion model in [3] and with mean reversion in [4]. Although Wong and Chan [2] approximate turbo prices under a stochastic volatility (SV) model, their solution is restricted to the assumption that the mean-reverting speed of the stochastic volatility should either be close to zero or to infinity. Our goal is to derive an analytic solution to turbo prices by relaxing such an assumption using a homotopy analysis method.

Homotopy analysis method was introduced by Ortega and Rheinboldt [5] in 1970 and has been applied to solve many nonlinear problems since the work of Liao [6] in 1992. Zhu in [7] pioneers the use of homotopy analysis method in financial mathematics and derives the first analytic formula to American options. Zhao and Wong in [8] show that the approach of Zhu is also applicable to general diffusion models.

While general diffusion models belong to the class of complete market models, SV models are of incomplete market models because the number of Brownian motions driving the asset dynamics is larger than one. Park and Kim [9] apply homotopy analysis method to solve vanilla option and barrier option prices under SV models. Leung [10] extends the framework to lookback option pricing. However, 
the application of homotopy analysis method to turbo warrant pricing under SV models is yet to be considered.

This paper employs the homotopy analysis method to solve the PDE for the turbo warrant price under a SV model. As the price of turbo warrant under the Black-Scholes model is available, we construct a homotopy which deforms from the Black-Scholes solution to the desired solution under the SV model. We highlight the fundamental challenge in turbo warrant pricing. A turbo warrant consists of a barrier option and a lookback rebate. Although the barrier option pricing under SV models is investigated in [9], Park and Kim do not consider rebate in knockout options. Our solution should cover the case of state-dependent rebate under SV. Second, Leung [10] offers an analytic pricing formula for lookback options but his formula cannot be directly used in calculating the lookback rebate, which is an expectation on the discounted lookback option with a random starting time. Therefore, our homotopy analysis method has to simultaneously solve these two problems together.

The reminder of this paper is organized as follows. Section 2 presents the nature of turbo warrants under SV models and the corresponding pricing problem in a PDE approach. Section 3 contains the main result of this paper and solves the PDE using homotopy analysis method. Concluding remark is made in Section 4.

\section{Problem Formulation}

Let $S_{t}$ be the underlying asset price at time $t$. A turbo call (put) warrant pays the contract holder $\left(S_{T}-K\right)^{+}$at maturity $T$ if a specified barrier $H \geq K$ has not been passed by $S_{t}$ at any time prior to the maturity. Denote $\tau_{H}$ as the first passage time that the asset price crosses the barrier $H$, that is, $\tau_{H}=$ $\inf \left\{t: S_{t} \leq H\right\}$. If $\tau_{H} \leq T$; then the contract is void and a new contract starts. The new contract is a call option on $m_{\tau_{H}}^{T_{0}}=\min _{\tau_{H} \leq t \leq \tau_{H}+T_{0}} S_{t}$, with the strike price $K$, and the time to maturity $T_{0}$. More precisely, the turbo call (TC) at time $t$ can be expressed as

$$
\begin{aligned}
\mathrm{TC}_{t}=E_{t}[ & e^{-r T}\left(S_{T}-K\right)^{+} \mathbf{1}_{\left\{\tau_{H}>T\right\}} \\
& \left.+e^{-r\left(\tau_{H}+T_{0}\right)}\left(m_{\tau_{H}}^{T_{0}}-K\right)^{+} \mathbf{1}_{\left\{\tau_{H} \leq T\right\}}\right],
\end{aligned}
$$

where $r$ is the constant interest rate and $E_{t}$ represents the riskneutral expectation given information up to time $t$. Wong and Chan [2] explain the incentive of this security design and show a model-free representation that the turbo call warrant is sum of a down-and-out call (DOC) and an expectation of a nonstandard lookback option (LB) starting at $\tau_{H}$.

Proposition 1 (see [2]). At $t \leq \tau_{H}$, the model-free representation of the turbo call warrant is

$$
T C(t, S)=\operatorname{DOC}(t, S)+E_{t}\left[e^{-r \tau_{H}} \mathbf{1}_{\left\{\tau_{H} \leq T\right\}} L B\left(\tau_{H}, S_{\tau_{H}}, T_{0}\right)\right],
$$

where DOC $(t, S)$ denotes the down-and-out call option price at $t$

$$
\begin{aligned}
L B\left(\tau_{H}, S_{\tau_{H}}, T_{0}\right)= & E_{\tau_{H}}\left[e^{-r T_{0}}\left(m_{\tau_{H}}^{T_{0}}-K\right)^{+} \mathbf{1}_{\left\{\tau_{H} \leq T\right\}}\right] \\
= & L C\left(0, S_{\tau_{H}}, \min \left(S_{\tau_{H}}, K\right), v_{\tau_{H}}, T_{0}\right) \\
& -\operatorname{LC}\left(0, S_{\tau_{H}}, S_{\tau_{H}}, v_{\tau_{H}}, T_{0}\right),
\end{aligned}
$$

and $L C\left(t, S, m, v, T_{0}\right)$ is the floating strike lookback call at time $t$ on $S$ with the realized minimum $m$, instantaneous volatility $v$, and time to maturity $T_{0}$.

In Proposition 1, the floating strike lookback call, $\mathrm{LC}\left(t, S, m, v, T_{0}\right)$, has the payoff

$$
S_{T_{0}}-\min _{0 \leq \tau \leq T_{0}}\left\{S_{\tau}\right\} .
$$

Hence, its price has the representation,

$$
\operatorname{LC}\left(t, S, m, v, T_{0}\right)=e^{-r\left(T_{0}-t\right)} E_{t}\left[S_{T_{0}}-\min _{0 \leq \tau \leq T_{0}}\left\{S_{\tau}\right\}\right] .
$$

The term $E_{t}\left[e^{-r \tau_{H}} \mathbf{1}_{\left\{\tau_{H} \leq T\right\}} \mathrm{LB}\left(\tau_{H}, S_{\tau_{H}}, T_{0}\right)\right]$ is called the downand-in lookback (DIL) option in [2] as a TC holder will knock in the lookback option LB, shown in (3), only when the underlying asset price hits the down-side barrier $H$.

In particular, if the asset price process follows the BlackScholes (BS) model where the volatility is a constant, then the turbo call price at $t \in\left[0, \tau_{H}\right]$ reduces to

$$
\begin{aligned}
\mathrm{TC}_{\mathrm{BS}}(t, S)= & \mathrm{DOC}_{\mathrm{BS}}(t, S) \\
+ & {\left[\mathrm{LC}_{\mathrm{BS}}\left(0, H, K, T_{0}\right)\right.} \\
& \left.\quad-\mathrm{LC}_{\mathrm{BS}}\left(0, H, H, T_{0}\right)\right] \mathrm{DR}_{\mathrm{BS}}(t, S),
\end{aligned}
$$

where the Black-Scholes pricing formulas of $\mathrm{DOC}_{\mathrm{BS}}, \mathrm{LC}_{\mathrm{BS}}$, and $\mathrm{DR}_{\mathrm{BS}}=E_{t}\left[e^{-r \tau_{H}} \mathbf{1}_{\left\{\tau_{H} \leq T\right\}}\right]$ are classical results and can be found in [11]. However, the formulas of $\mathrm{LC}_{\mathrm{BS}}$ and $\mathrm{DR}_{\mathrm{BS}}$ also appear in some proofs of this paper. Specifically, $\mathrm{LC}_{\mathrm{BS}}\left(t, S, K, T_{0}\right)=S \bar{U}_{0}\left(t, y, v, T_{0}\right)$, where $\bar{U}_{0}$ is given by (19) while $\mathrm{DR}_{\mathrm{BS}}$ appears in (28).

2.1. Turbos under Stochastic Volatility. Although the explicit BS formula for the TC is known as in (6), its analytic pricing formula under SV model is yet to be considered. The Heston SV model assumes the following stochastic differential equation (SDE) for the underlying asset price

$$
\frac{d S_{t}}{S_{t}}=(r-q) d t+\sqrt{v_{t}} d W_{t}^{S}
$$

where $r$ is the constant interest rate, $q$ is the constant dividend yield, and $v_{t}$ is the stochastic instantaneous variance of the asset. The stochastic variance $v_{t}$ follows the SDE:

$$
d v_{t}=\kappa\left(\theta-v_{t}\right) d t+\sigma \sqrt{v_{t}} d W_{t}^{v}
$$

where $\kappa, \theta$, and $\sigma$ are constants. In (7) and (8), $W_{t}^{S}$ and $W_{t}^{v}$ are Wiener processes defined on a filtered complete probability 
space $\left(\Omega, \mathscr{F}, \mathcal{Q}, \mathscr{F}_{t \geq 0}\right)$ with $E\left[W_{t}^{S} W_{t}^{v}\right]=\rho t$, where $\mathcal{Q}$ denotes the risk-neutral probability measure.

Let $\widetilde{V}(t, S, v)$ be the turbo warrant price at time $t$ under the stochastic volatility $v$. Using (1), we have

$\widetilde{V}(t, S, v)$

$$
\begin{aligned}
=E[ & e^{-r T}\left(S_{T}-K\right)^{+} \mathbf{1}_{\left\{\tau_{H}>T\right\}} \\
& \left.\quad+e^{-r\left(\tau_{H}+T_{0}\right)}\left(m_{\tau_{H}}^{T_{0}}-K\right)^{+} \mathbf{1}_{\left\{\tau_{H} \leq T\right\}} \mid S_{t}=S, v_{t}=v\right] .
\end{aligned}
$$

By Proposition 1, the turbo price can be expressed as (2). As the SV model assumes a continuous process for the underlying asset price, $S_{\tau_{H}}=H$. Hence, we have

$$
\begin{aligned}
& \widetilde{V}(t, S, v) \\
& =E\left[e^{-r T}\left(S_{T}-K\right)^{+} \mathbf{1}_{\left\{\tau_{H}>T\right\}}\right. \\
& \left.+e^{-r \tau_{H}} \mathbf{1}_{\left\{\tau_{H} \leq T\right\}} \operatorname{LB}\left(\tau_{H}, H, v_{\tau_{H}}, T_{0}\right) \mid S_{t}=S, v_{t}=v\right] \text {, }
\end{aligned}
$$

where

$$
\begin{aligned}
\operatorname{LB}\left(\tau_{H}, H, v_{\tau_{H}}, T_{0}\right)= & \operatorname{LC}\left(0, H, K, v_{\tau_{H}}, T_{0}\right) \\
& -\operatorname{LC}\left(0, H, H, v_{\tau_{H}}, T_{0}\right) .
\end{aligned}
$$
$x_{t}$ as

Let $x_{t}=\ln \left(S_{t} / H\right)$. By Ito's lemma, we obtain the SDE for

$$
d x_{t}=\left(r-q-\frac{v_{t}}{2}\right)+\sqrt{v_{t}} d W_{t}^{S}, \quad x_{0}=\ln \left(\frac{S_{0}}{H}\right) .
$$

Let $V(t, x, v)=\widetilde{V}(t, S, v)$. Applying the Feynman-Kač formula to $V$ in (10) with respect to (12) and (8), we have

$$
\begin{gathered}
(\mathscr{L}+\mathscr{M}) V=0 \\
V(T, x, v)=\left(H e^{x}-K\right)^{+} \\
\left.V(t, x, v)\right|_{x=0}=\operatorname{LB}\left(t, H, v, T_{0}\right),
\end{gathered}
$$

where

$$
\begin{aligned}
& \mathscr{L}=\frac{\partial}{\partial t}+\left(r-q-\frac{v}{2}\right) \frac{\partial}{\partial x}+\frac{1}{2} v \frac{\partial^{2}}{\partial x^{2}}-r, \\
& \mathscr{M}=\kappa(\theta-v) \frac{\partial}{\partial v}+\rho \sigma v \frac{\partial^{2}}{\partial x \partial v}+\frac{1}{2} \sigma^{2} v \frac{\partial^{2}}{\partial v^{2}} .
\end{aligned}
$$

In addition, the rebate LB is related to LC through (11). Using the transformation of variable $\mathrm{LC}(t, S, m, v, T)=S$. $U(t, \log (m / S), v, T)$, the function $U$ is the solution of the following BVP [10]:

$$
\begin{gathered}
(\mathscr{L}+\mathscr{M}) U=0 \\
U(T, x, v, T)=e^{x}-1 \\
\left.\frac{\partial U(t, x, v, T)}{\partial x}\right|_{x=0}=0 .
\end{gathered}
$$

In principal, the exotic lookback option $\mathrm{LB}(t, S, v, T)$ should be solved from an alternative PDE governing lookback options. Fortunately, we know from (11) that this exotic lookback option is the difference between two floating strike lookback calls.

Specifically, $\operatorname{LC}(t, S, m, v, T)$ has the following homotopy expression [10]:

$$
\mathrm{LC}(t, S, m, v, T)=\left.S \cdot \bar{U}(t, y, v, T, p)\right|_{p=1},
$$

where $y=\ln (m / S)$;

$$
\bar{U}(t, y, v, T, p)=\sum_{n=0}^{\infty} \bar{U}_{n}(t, y, v, T) p^{n}
$$

The functions $\bar{U}_{n}(t, y, v, T), n=1,2, \ldots$, are obtained from the following iteration:

$$
\begin{gathered}
\bar{U}_{n}(t, y, v, T)=\eta(t, y, v) \widehat{U}_{n}(t, y, v, T), \\
\eta(\tau, y, v)=\exp \left\{\left(-q-\frac{1}{8} v(\alpha(v)+1)^{2}\right) \tau+\frac{1}{2}(\alpha(v)+1) x\right\}, \\
\alpha(v)=\frac{2(r-q)}{v}, \quad k=\frac{1}{2}(\alpha(v)+1), \\
\widehat{U}_{n}(t, y, v, T)=\int_{0}^{\tau} \int_{0}^{\infty} \eta(s, \xi, v) \mathfrak{M} \bar{U}_{n-1}(T-s, \xi, v, T) \\
\mathfrak{M}=\rho \sigma v \frac{\partial}{\partial v}-\rho \sigma v \frac{\partial}{\partial y \partial v}+\kappa(\theta-v) \frac{\partial}{\partial v}+\frac{1}{2} \sigma^{2} v \frac{\partial^{2}}{\partial v^{2}}, \\
\bar{G}(t, y, \xi, v)=\frac{1}{\sqrt{2 \pi v t}}\left\{e^{-(y-\xi)^{2} / 2 v t}+e^{-(y+\xi)^{2} / 2 v t}, y, \xi, v\right) d \xi d s, \\
\left.+2 k \int_{0}^{\infty} e^{-\left((y+\xi+\eta)^{2} / 2 v t\right)+k \eta} d \eta\right\}
\end{gathered}
$$

where the iteration begins with the function

$$
\begin{gathered}
\bar{U}_{0}(t, y, v, T)=e^{y-r(T-t)} N\left(-d_{m}^{-}\right)-e^{-q(T-t)} N\left(-d_{m}^{+}\right) \\
+\frac{v}{2(r-q)}\left[e^{-q(T-t)} N\left(d_{m}^{+}\right)\right. \\
\left.-e^{-r(T-t)+(y(r-q) / v)} N\left(d_{m}^{r}\right)\right] \\
d_{m}^{ \pm}=\frac{-y+(r-q \pm(v / 2))(T-t)}{\sqrt{v(T-t)}} \\
d_{m}^{r}=d_{m}^{+}-2(r-q) \sqrt{\frac{T-t}{v}}
\end{gathered}
$$

with $N(\cdot)$ being the standard normal cumulative distribution function. 


\section{The Homotopy Framework}

We aim to construct a homotopy solution $\bar{V}(t, x, v, p)$ with auxiliary parameter $p \in[0,1]$ such that $\bar{V}(t, x, v, 0)=$ $V_{0}(t, x, v)$ and $\bar{V}(t, x, v, 1)=V(t, x, v)$, the desired pricing formula. The function $V_{0}$ can be regarded as the initial guess of the solution. We use the BS formula of TC in (6) as the initial guess. Therefore, $V_{0}(t, x, v)$ is the solution of the following PDE:

$$
\begin{gathered}
\mathscr{L} V_{0}(t, x, v)=0 \\
V_{0}(T, x, v)=\left(H e^{x}-K\right)^{+} \\
\left.V_{0}(t, x, v)\right|_{x=0}=H\left[U_{0}\left(0,0, v, T_{0}\right)-U_{0}\left(0, \ln \frac{K}{H}, v, T_{0}\right)\right],
\end{gathered}
$$

where $\mathscr{L}$ is defined in (14) and $U_{0}$ in (19).

Consider the following PDE for $\bar{V}(t, x, v, p)$ :

$$
\begin{gathered}
-p(\mathscr{L}+\mathscr{M}) \bar{V}=(1-p) \mathscr{L}\left(\bar{V}-V_{0}\right) \\
\bar{V}(T, x, v, p)=\left(H e^{x}-K\right)^{+} \\
\left.\bar{V}(t, x, v, p)\right|_{x=0}=H\left[\bar{U}\left(0,0, v, T_{0}, p\right)-\bar{U}\left(0, \ln \frac{K}{H}, v, T_{0}, p\right)\right],
\end{gathered}
$$

where $x=\ln (S / H), \bar{U}$ is defined in (17), and $\mathscr{L}$ and $\mathscr{M}$ are defined in (14).

If we set $p=1$ in (22), then we have

$$
\begin{gathered}
(\mathscr{L}+\mathscr{M}) \bar{V}=0 \\
\bar{V}(T, x, v, 1)=\left(H e^{x}-K\right)^{+} \\
\left.\bar{V}(t, x, v, 1)\right|_{x=0}=H\left[\bar{U}\left(0,0, v, T_{0}, 1\right)-\bar{U}\left(0, \ln \frac{K}{H}, v, T_{0}, 1\right)\right] \\
=\operatorname{LC}\left(H, H, v, T_{0}\right)-\operatorname{LC}\left(H, K, v, T_{0}\right) \\
=\operatorname{LB}\left(t, H, v, T_{0}\right) .
\end{gathered}
$$

By comparing the systems (13) and (23), we confirm that $\bar{V}(t, x, v, 1)=V(t, x, v)$.

Alternatively, setting $p=0$ in (22) yields

$$
\begin{aligned}
\mathscr{L} \bar{V}(t, x, v, 0)=\mathscr{L} V_{0}(t, x, v) \\
\bar{V}(T, x, v, 0)=\left(H e^{x}-K\right)^{+} \\
\left.\bar{V}(t, x, v, 0)\right|_{x=0}=H\left[\bar{U}\left(0,0, v, T_{0}, 0\right)-\bar{U}\left(0, \ln \frac{K}{H}, v, T_{0}, 0\right)\right] \\
=H\left[U_{0}\left(0,0, v, T_{0}\right)-U_{0}\left(0, \ln \frac{K}{H}, v, T_{0}\right)\right],
\end{aligned}
$$

where $U_{0}\left(0, y, v, T_{0}\right)$ is obtained in (19). Therefore, $\bar{V}(t, x, v, 0)=V_{0}(t, x, v)$. Hence, the PDE (22) is desired construction for the homotopy solution.
Consider the Taylor expansion of $\bar{V}(t, x, v, p)$ with respect to $p$ as follows:

$$
\bar{V}(t, x, v, p)=\sum_{n=0}^{\infty} \bar{V}_{n}(t, x, v) p^{n},
$$

where $\bar{V}_{n}(t, x, v)=\left.(1 / n !)\left(\partial^{n} / \partial p^{n}\right) \bar{V}(t, x, v, p)\right|_{p=0}$. Substituting (25) into (22) yields

$$
\begin{gathered}
\mathscr{L} \bar{V}_{n}(t, x, v)+\mathscr{M} \bar{V}_{n-1}(t, x, v)=0, \\
\bar{V}_{n}(T, x, v)=0, \\
\left.\bar{V}_{n}(t, x, v)\right|_{x=0}=H\left[\bar{U}_{n}\left(0,0, v, T_{0}\right)-\bar{U}_{n}\left(0, \ln \frac{K}{H}, v, T_{0}\right)\right], \\
\text { for } n=1,2, \ldots,
\end{gathered}
$$

where $\bar{U}_{n}\left(0, y, v, T_{0}\right)$ is a known function given in (19). Let $\tau=T-t$. Then the PDE (26) becomes, for $n=1,2, \ldots$,

$$
\begin{gathered}
\frac{\partial \bar{V}_{n}}{\partial \tau}=\frac{1}{2} v \frac{\partial^{2} \bar{V}_{n}}{\partial x^{2}}+\left(r-q-\frac{v}{2}\right) \frac{\partial \bar{V}_{n}}{\partial x}-r \bar{V}_{n}+M \bar{V}_{n-1} \\
\bar{V}_{n}(T, x, v)=0 \\
\left.\bar{V}_{n}(\tau, x, v)\right|_{x=0}=H\left[\bar{U}_{n}\left(0,0, v, T_{0}\right)\right. \\
\left.-\bar{U}_{n}\left(0, \ln \frac{K}{H}, v, T_{0}\right)\right]:=H_{n}^{*}(v) .
\end{gathered}
$$

This PDE can be transformed into a heat equation and its solution has a classical result. Specifically, the result is summarized as follows.

Theorem 2. Suppose the underlying asset price, $S_{t}$, follows the $S V$ model of (7) and (8). Then, the turbo call warrant price in (1) has an analytic formula derived from the iteration: for $n=1,2, \ldots$,

$$
\begin{aligned}
& \bar{V}_{n}(\tau, x, v)= H_{n}^{*}(v) D R_{B S}(\tau, x, v) \\
&+\int_{0}^{\tau} \int_{0}^{\infty} \mathscr{M} \bar{V}_{n-1}(s, x, v) G(\tau-s, x, \xi, v) d \xi d s \\
& D R_{B S}(\tau, x, v)=\left.\frac{v}{2} \int_{0}^{\tau} \frac{\partial}{\partial \xi} G(\tau-s, x, \xi, v)\right|_{\xi=0} d s ; \\
& G(t, x, \xi, v)= \frac{1}{\sqrt{2 \pi v t}} e^{((r-q-(v / 2))(\xi-x) / v)-\left(r+\left((r-q-(v / 2))^{2} / 2 v\right)\right) t} \\
& \times\left(e^{-(x-\xi)^{2} / 2 v t}-e^{-(x+\xi)^{2} / 2 v t}\right) .
\end{aligned}
$$

An analytic pricing formula of TC under SV model is then given by

$$
V(t, x, v)=\bar{V}(\tau, x, v, 1)=\sum_{n=0}^{\infty} \bar{V}_{n}(\tau, x, v)
$$


3.1. DOC and DIL. As the TC price paying a lookback rebate is a sum of the DOC and DIL prices, the solution of $\bar{V}(t, x, v, p)$ from (25) and (28) is useful to determine the DOC option price and DIL option price under SV. Hence, our solution also contributes to the valuation of barrier-type options under SV model. Note that the N-CBBC is actually a DOC option.

For DOC option with barrier level $H$, the corresponding PDE is the same as (13) except that the boundary condition is replaced by $\left.V(t, x, v)\right|_{x=0}=0$. Using (28) and (29), we immediately obtain that

$$
\operatorname{DOC}(t, x, v, T)=\sum_{n=0}^{\infty} \overline{\operatorname{DOC}}_{n}(\tau, x, v),
$$

where

$$
\begin{aligned}
& \overline{\mathrm{DOC}}_{n}(\tau, x, v)=\int_{0}^{\tau} \int_{0}^{\infty} \mathscr{M} \overline{\mathrm{DOC}}_{n-1}(s, x, v) \\
& \times G(\tau-s, x, \xi, v) d \xi d s, \\
& \overline{\mathrm{DOC}}_{0}(\tau, x, v)=\mathrm{DOC}_{\mathrm{BS}}(t, x, v, T) .
\end{aligned}
$$

Here, we see that the DOC price under SV model from our approach is consistent with the result by Park and Kim [9].

For DIL option with barrier level $H$, the corresponding $\mathrm{PDE}$ is the same as (13) except that the terminal condition is replaced by $V(T, x, v)=0$. Using (28) and (29), we obtain that

$$
\operatorname{DIL}(t, x, v, T)=\sum_{n=0}^{\infty} \overline{\operatorname{DIL}}_{n}(\tau, x, v),
$$

where

$$
\begin{gathered}
\overline{\mathrm{DIL}}_{n}(\tau, x, v)=H_{n}^{*}(v) \mathrm{DR}_{\mathrm{BS}}(\tau, x, v) \\
+\int_{0}^{\tau} \int_{0}^{\infty} M \overline{\mathrm{DIL}}_{n-1}(s, x, v) \\
\times G(\tau-s, x, \xi, v) d \xi d s, \\
\overline{\mathrm{DIL}}_{0}(\tau, x, v)=H_{0}^{*}(v) \mathrm{DR}_{\mathrm{BS}}(\tau, x, v) .
\end{gathered}
$$

\section{Conclusion}

We use a PDE approach to solve the price of turbo warrant under a SV model. The PDE is solved by means of homotopy analysis method. The boundary condition of the PDE is simplified using the homotopy solution developed in [10]. As byproducts, we offer analytic pricing formulas for DOC and DIL options under SV model. Future research can apply this solution to investigate the impact of volatility to the prices of turbo warrants empirically.

\section{Acknowledgments}

H. Y. Wong acknowledges the support by GRF of Research Grant Council of Hong Kong with Project n. 403511. M. C. Chiu acknowledges the Start-up Research Grant RG44/20122013R by Department of MIT, Hong Kong Institute of Education.

\section{References}

[1] The HKEx callable bull/bear contract (CBBC) market, Report by Research \& Corporate Development Department of Hong Kong Exchanges and Clearing Limited, 2009, http://www.hkex .com.hk/eng/stat/research/rpaper/Documents/cbbc2009.pdf.

[2] H. Y. Wong and C. M. Chan, "Turbo warrants under stochastic volatility," Quantitative Finance, vol. 8, no. 7, pp. 739-751, 2008.

[3] H. Y. Wong and K. Y. Lau, "Analytical valuation of turbo warrants under double exponential jump diffusion," Journal of Derivatives, pp. 61-73, 2008.

[4] H. Y. Wong and K. Y. Lau, "Path-dependent currency options with mean reversion," The Journal of Futures Markets, vol. 28, pp. 275-293, 2008.

[5] J. M. Ortega and W. C. Rheinboldt, Iterative Solution of Nonlinear Equations in Several Variables, Academic Press, New York, NY, USA, 1970.

[6] S. J. Liao, The proposed homotopy analysis technique for the solution of nonlinear problems [Ph.D. thesis], Shanghai Jiao Tong University, 1992.

[7] S.-P. Zhu, "An exact and explicit solution for the valuation of American put options," Quantitative Finance, vol. 6, no. 3, pp. 229-242, 2006.

[8] J. Zhao and H. Y. Wong, "A closed-form solution to American options under general diffusion processes," Quantitative Finance, vol. 12, no. 5, pp. 725-737, 2012.

[9] S.-H. Park and J.-H. Kim, "Homotopy analysis method for option pricing under stochastic volatility," Applied Mathematics Letters, vol. 24, no. 10, pp. 1740-1744, 2011.

[10] K. S. Leung, "An analytic pricing formula to lookback options under stochastic volatility," Applied Mathematics Letters, vol. 26, pp. 145-149, 2013.

[11] Y.-K. Kwok, Mathematical Models of Financial Derivatives, Springer, Berlin, Germany, 2008. 


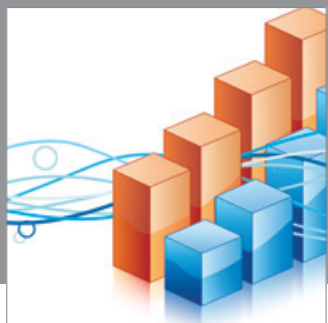

Advances in

Operations Research

mansans

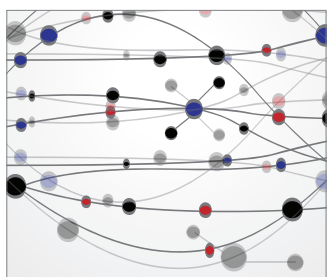

The Scientific World Journal
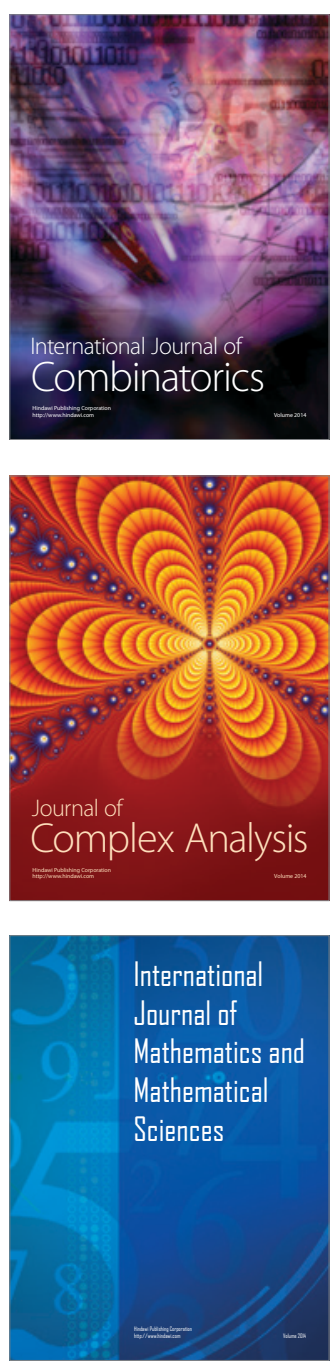
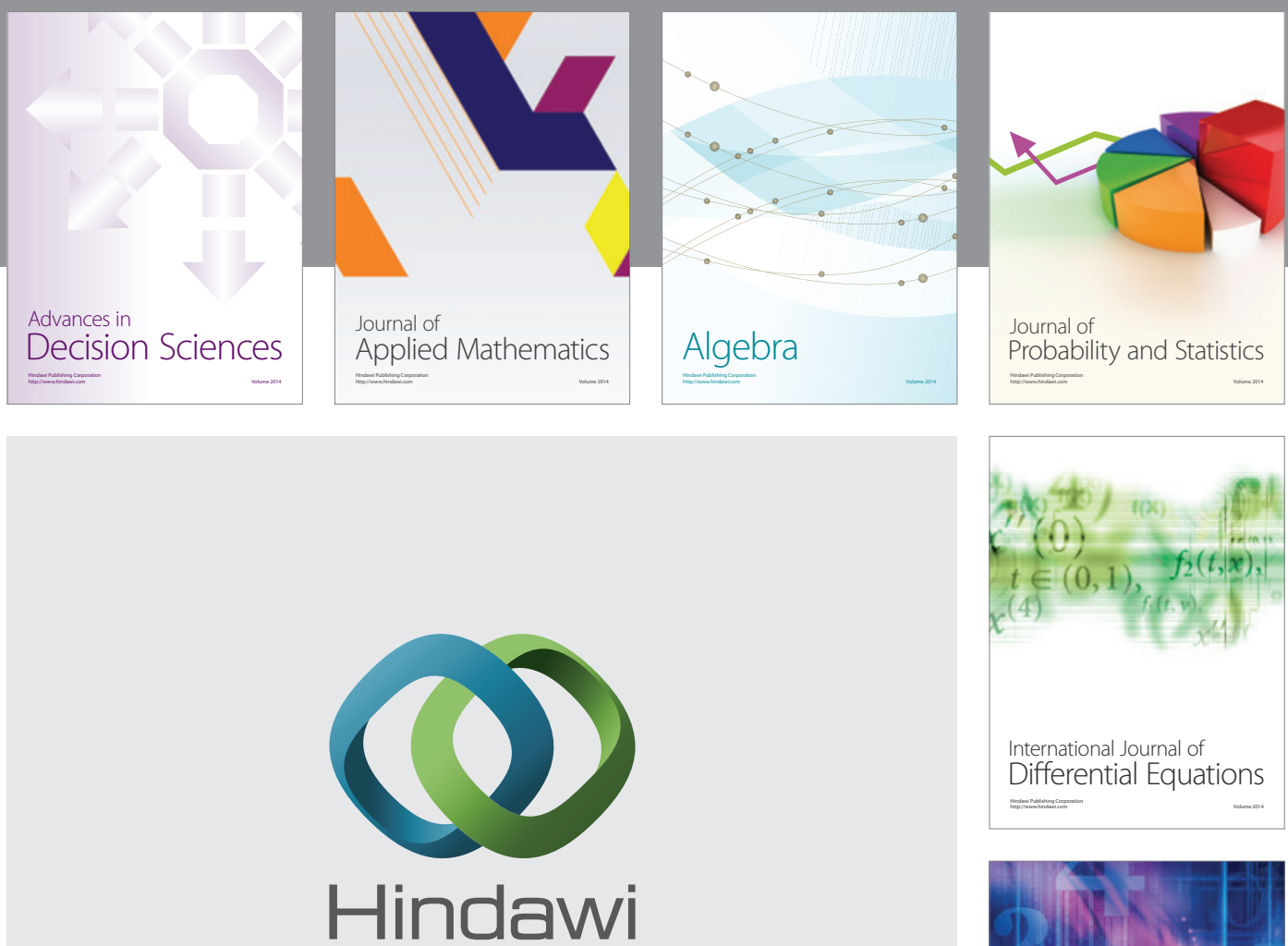

Submit your manuscripts at http://www.hindawi.com
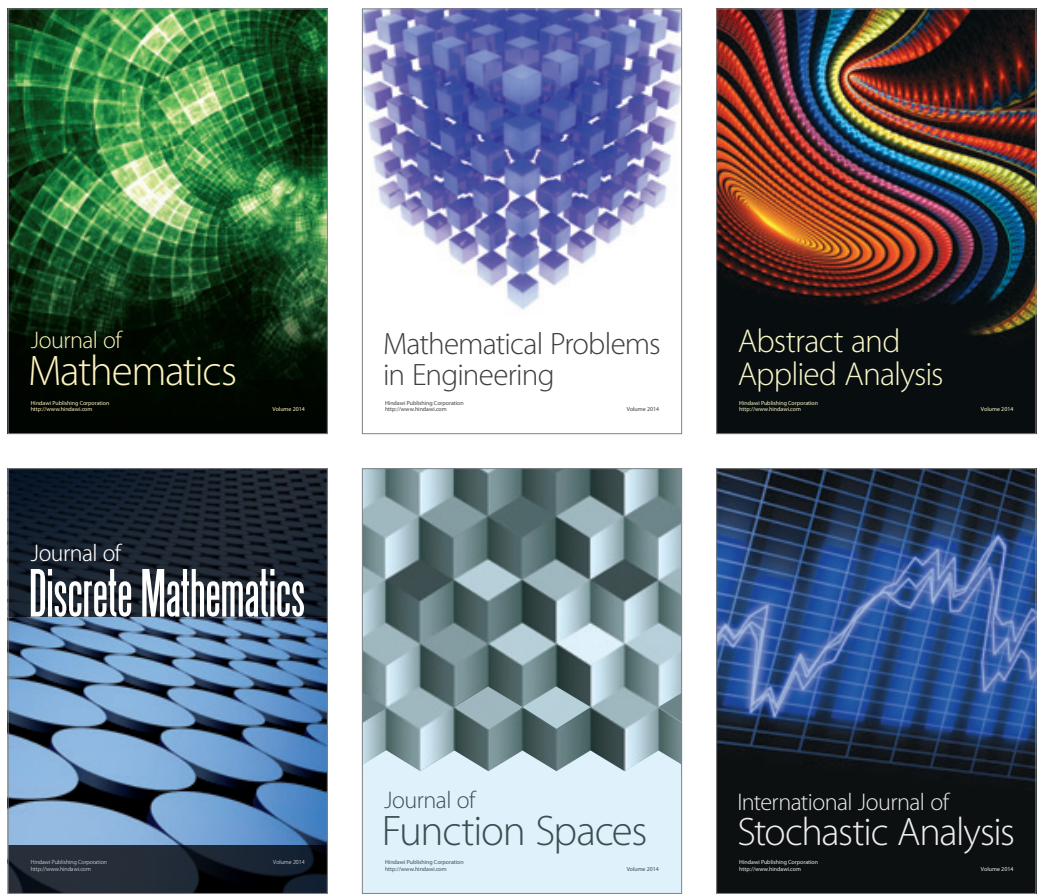

Journal of

Function Spaces

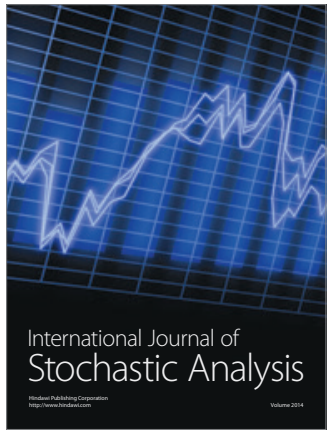

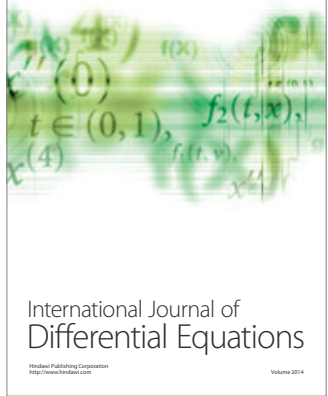
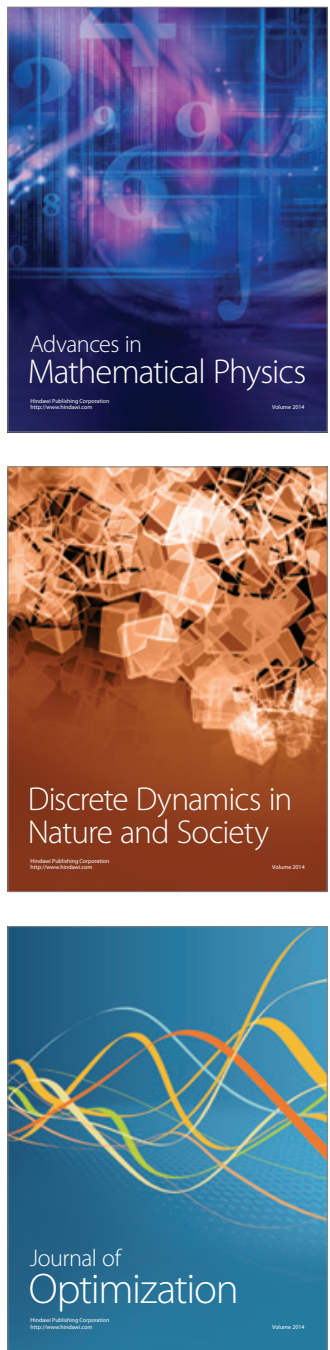